\title{
Color doppler as a valuable procedure for early diagnosis of ocular vascular changes in Behcet's disease
}

\begin{abstract}
This study was performed to study the role of Color Doppler Ultrasound (CDU) in hemodynamic evaluation of the orbital vessels in patients with Behçet's disease.

Thirty patients suffering from Behçet's disease were included in this study satisfying at least 3 of the criteria of BD according to the International Study Group for Behçet's disease in 1990, in addition to fifteen normal healthy individuals of matched age and sex who served as controls.

They were subjected to full history taking, full clinical examination and routine laboratory investigations together with full ophthalmological examination. Color Doppler Ultrasound (CDU) was done to evaluate the blood flow of the orbital vessels.

There were diminished blood flow velocities of the Ophthalmic Artery (OA), Central Retinal Artery (CRA) and Posterior Ciliary Artery (PSA), as peak systolic velocities (PSV) and end diastolic velocities (EDV) of these arteries were lower than normal and resistance flow indices (RI) were higher than normal.

We concluded that patients with Behçet disease and ocular involvement have lower CRA, PCA and OA blood flow velocities than healthy volunteers. Early diagnosis of ocular involvement is important to start appropriate treatment and therefore delay disease progression. CDU has taken its place as a widely used, easy-to-perform, feasible, simple, relatively low-cost, rapid, accurate, and noninvasive imaging method for evaluating ocular hemodynamics.
\end{abstract}

Keywords: behcet disease, color doppler ultrasound
Volume 10 Issue 3 - 2018

\author{
H H Al Sherbeni,' S Metawie,' R Abdel \\ salam, ${ }^{2} \mathrm{H}$ Azizi ${ }^{3}$ \\ 'Rheumatology and Rehabilitation, Faculty of Medicine- Cairo \\ University, Egypt \\ ${ }^{2}$ Ophthalmology, Faculty of Medicine, Cairo University, Egypt \\ ${ }^{3}$ Radiology, Faculty of Medicine, Cairo University, Egypt
}

Correspondence: $\mathrm{HH}$ Al Sherbeni, Rheumatology and Rehabilitation, Faculty of Medicine- Cairo University, Egypt, Email hhalsherbeni@gmail.com

Received: Jan 17,2018| Published: June 04, 2018

\section{Background}

Ocular involvement is a common and serious manifestation of Behçet's disease (BD). It occurs in about $70 \%$ of the patients and is associated with a high risk of blindness. ${ }^{1}$ Color Doppler is used for imaging retro-bulbar vasculature. It measures the peak systolic velocity (PSV) and end diastolic velocity (EDV), from which the resistance index (RI) is calculated in the ophthalmic artery (OA), central retinal artery (CRA), and posterior ciliary artery (PCA) to detect any abnormality in the retrobulbar vessels. ${ }^{2}$

\section{Objectives}

To determine and compare the orbital Color Doppler hemodynamic parameters of Behçet's patients with those of healthy subjects and to evaluate the OA, CRA, PCA flow velocities and resistance indices (RIs).

\section{Patients \& methods}

This study included thirty patients, 28 males and 2 females, suffering from Behcet's disease satisfying at least 3 of the criteria of $\mathrm{BD}$ according to the International Study Group for Behçet's disease in $1990 .^{3}$

They were collected from the Rheumatology and Rehabilitation department, Cairo University Hospitals.
Their ages ranged from 18 to 45 years. Their disease duration ranged from 0.25 to 20 years.

Fifteen healthy volunteers ( 13 males and 2 females) were also included in this study. Their ages ranged from 18 to 43 years.

In this study, Patients with hypertension, diabetes mellitus, cerebrovascular diseases, and peripheral vascular diseases were excluded. All patients were diagnosed according to the International Study Group for Behçet's disease in $1990 .^{3}$

All patients and controls were subjected to full history taking, complete physical examination and laboratory investigations.

The patients were subjected to full ophthalmological examination at the ophthalmology department. This included examination of visual acuity by snellen chart which measure visual acuity at a distance 6 meters and range from $6 / 60$ up to $6 / 6$. If the patient couldn't see, a counting fingers test was done to a distance from $100 \mathrm{~cm}$ to $50 \mathrm{~cm}$. If he did not see a hand movement test was done and if not, light perception was done.

A routine slit lamp bio microscopy examination was done to examine anterior chamber of the eye, cornea, lens and vitreous.

Examination with assisting lens (auxiliary lens +90D) was done to examine the posterior chamber (posterior pole of the retina, optic nerve and macula). 
Examination during fundus dilatation with mydracyl (tropicamide) eye drops to examine a wide field of the retina.

Assessment of disease activity of all patients using the Behçet's Disease current Activity Form (BDCAF): The Behçet's Disease current Activity Form (BDCAF), which offers an easy, complete and reliable method of assessing and documenting clinical activity in patients with BD was performed to our patients Table $1 .^{4}$

Scoring system for activity form:All scoring depends on the symptoms present over the preceding the four weeks prior to assessment. Only clinical features that the clinician feels are due to Behçet's disease should be scored.

Scoring for fatigue, headache, oral and genital ulceration, skin lesion, joint symptoms and gastrointestinal symptoms

It is based on duration of symptoms (round up to nearest week). The following question is asked and the blank is filled in with the organ system assessed. "Over the last 4 weeks, for how weeks in total you had ...........?."

0-- No symptoms

1-- Symptoms for 1 week

2-- Symptoms for 2 weeks

3-- Symptoms for 3 weeks

4-- Symptoms for 4 weeks

\section{Eye Involvement}

The following question is asked (ticked if symptoms are present):

"Over the last 4 weeks have you had a red eye, a painful eye, or blurred or reduced vision?"

In the presence of any of these symptoms or if the physician feels there is any activity, the patient is referred to an ophthalmologist to determine the eye score (Behçet's Oculopathy Index).

\section{Nervous system}

The following question is asked (ticked if symptom is present):

"Over the last 4 weeks have you had any blackout, difficulty with speech or hearing, double vision, weakness or loss of feeling of the face, arm or leg, memory loss or loss of balance?"

If the answer to this question is "no" then answers to questions 1-5 are deemed negative, otherwise determine the following:

Q1: Are there new symptoms or signs consistent with meningeal involvement?

Q2: Are there new symptoms or signs consistent with isolated cranial nerve involvement?

Q3: Are there new symptoms or signs consistent with brain system or cerebellar involvement?

Q4: Are there new symptoms or signs consistent with cerebral hemisphere involvement?

Q5: Are there new symptoms or signs consistent with spinal cord involvement?

\section{Major vessel involvement (exclude neurological involvement)}

The following question is asked (ticked if symptom is present):

"Over the last 4 weeks have you had chest pain, breathlessness, coughed up blood, or had any pain, swelling or discoloration of either of the face, arm or leg?"

If the answer to this question is "no" then answers to questions 1-4 are deemed negative, otherwise determine the following:

Q1: Are there new symptoms or signs consistent with peripheral deep venous thrombosis?

Q2: Are there new symptoms or signs consistent with central deep venous thrombosis?

Q3: Are there new symptoms or signs consistent with peripheral arterial thrombosis /aneurysm?

Q4: Are there new symptoms or signs consistent with pulmonary arterial thrombosis /aneurysm? ${ }^{4}$

\section{The averge mean (BDCAF) score}

Reflecting disease activity over time will be calculated by summating score of every patient and then dividing the total by number of patients. ${ }^{4}$

\section{Radiological investigation}

Hemodynamic evaluation of orbital vessels was carried out by Color Doppler ultrasound (CDU) with ATL Phillips 5000 (USA) Doppler Ultrasonography device by using a 7.5-MHz linear probe.

For motion toward the probe, the code is red-orange yellow and for motion away from the probe the code is blue. Green is used for abnormal high velocities which indicate turbulence. ${ }^{5}$

Sound waves are sent from the probe at a given frequency $7.5 \mathrm{MHz}$, and blood flow velocity is determined by the shift in the frequency of the returning sound waves. ${ }^{6}$

Flow velocity data is graphed against time. The peak and trough of the wave are identified by the operator. From these, the computer calculates peak systolic (PSV), end diastolic (EDV) velocities and resistance index (RI).

The use of CDU to measure blood flow parameters in retro bulbar vessels has several advantages compared to other technologies. As it can provide information about the flow, direction, course, and position of blood vessels and allowing hemodynamic data to be obtained in eyes with poor optical media like cataract, vitreous hemorrhages, synechia, and small pupil. ${ }^{5}$

\section{The technique}

The patient lies down in supine position and the probe is placed on the closed eyelid after application of sterile ultrasound coupling gel. This was performed by an experienced radiologist. During the CDU examination, any pressure on the eye was avoided.

Linear or sectorial ultrasound with frequencies 7.5 was used and transverse or sagittal sections through orbit were obtained. The operator identified the desired vessel based on anatomical location.

The velocity curves are graphed by the device: velocity on Y- axis against time on the X-axis. The velocity wave form differs between vessels, and may be used to confirm the vessel being inspected. Then, 
the radiologist identifies the peak systolic and end diastolic velocities from the velocity wave form and resistivity index of the ophthalmic artery (OA), central retinal artery (CRA), posterior ciliary artery (PCA) are automatically calculated by the device.

\section{Ophthalmic artery}

Normal value for

PSV is $(30 \pm 4)$.

EDV is $(11.2 \pm 3.7)$.

$\mathrm{RI}$ is $(0.75)$.

II. Central retinal artery
Normal value for

PSV is $(12 \pm 2)$.

EDV is (3.7 \pm 0.9$)$.

RI is $(0.72)$.

III. Posterior ciliary arteries

Normal value for

PSV is $(10-12 \pm 4)$.

$\mathrm{EDV}$ is $(5.7 \pm 1.5)$.

$\mathrm{RI}$ is $(0.68)$

Table I The Behçet's disease current activity form (BDCAF)

\begin{tabular}{|c|c|c|c|c|c|c|}
\hline Clinical feature & Activity & & & & & Other clinical details \\
\hline Fatigue & 0 & 1 & 2 & 3 & 4 & \\
\hline Headache & 0 & 1 & 2 & 3 & 4 & \\
\hline Oral ulceration & 0 & 1 & 2 & 3 & 4 & \\
\hline Skin lesions: Erythema nod osum or superficial thrombophlebitis & 0 & 1 & 2 & 3 & 4 & \\
\hline Joints: Arthralgia & 0 & 1 & 2 & 3 & 4 & \\
\hline arthritis & 0 & 1 & 2 & 3 & 4 & \\
\hline GIT: nausea or vomiting or abdominal pain & 0 & 1 & 2 & 3 & 4 & \\
\hline & 0 & 1 & 2 & 3 & 4 & \\
\hline Eye:is there eye activity? & 0 & 1 & & & & \\
\hline Behçet's Oculopathy index (completed by ophthalmologist) & 0 & 1 & 2 & 3 & & \\
\hline & 0 & 1 & \multicolumn{3}{|c|}{ Ask questions below } & \\
\hline $\begin{array}{l}\text { CNS is there new nervous system activity if yes, answer questions } \\
\text { below }\end{array}$ & $\begin{array}{l}\text { Q1 } \\
\text { Yes } \\
\text { /No }\end{array}$ & $\begin{array}{l}\text { Q2 } \\
\text { Yes } \\
\text { /No }\end{array}$ & $\begin{array}{l}\text { Q3 } \\
\text { Yes } \\
\text { /No }\end{array}$ & $\begin{array}{l}\text { Q4 } \\
\text { Yes } \\
\text { /No }\end{array}$ & $\begin{array}{l}\text { Q5 } \\
\text { Yes } \\
\text { /No }\end{array}$ & \\
\hline \multirow{3}{*}{$\begin{array}{l}\text { Major vessel is there new major vessel activity? } \\
\text { If yes answer questions below }\end{array}$} & 0 & 1 & \multicolumn{2}{|c|}{ Ask questions below } & & \\
\hline & Q1 & $\mathrm{Q} 2$ & Q3 & Q4 & & \\
\hline & Yes/ No & $\begin{array}{l}\text { Yes/ } \\
\text { No }\end{array}$ & Yes/ No & Yes/ No & & \\
\hline
\end{tabular}

\section{Statistical analysis}

An IBM compatible personal computer was used to store and analyze the data and to produce graphic presentation of important results. Calculation were done by means of statistical software package namely, SPSS. Date were tabulated and statistically analyzed to evaluate the difference between the groups under study as regards the various parameters. Together, correlations were tried in-between the essential studied parameters. The statistical analysis includes:

Range.

The arithmetic mean.

Standard deviation.

Hypothesis student's (t) test.

Pearson's correlation coefficient.

\section{Significance of results was calculated as follows}

Non-significant (NS) if $\mathrm{P}>0.05$.

Significant ( $\mathrm{S}$ ) if $\mathrm{P}<0.05$.

Highly significant (HS) if $\mathrm{P}<0.01$.

\section{Results}

This study included 30 patient's 28 (93\%) males and $2(7 \%)$ females with Behçet disease fulfilling the criteria of the International Study Group for classification of BD in $1990 .{ }^{3}$ Their age ranged from 18 to 45 years with a mean of $31.0^{ \pm} 7.5$. Their disease duration ranged from 0.25 to 20 years with a mean of $5.71 \pm 5.84$. Patient index score ranged from 0 to 7.0 with a mean of $3.27 \pm 1.53$. Fifteen healthy adults served as controls $14(93 \%)$ males and $1(7 \%)$ female. Their ages range from 18 to 43 years with a mean of $30 \pm 5.48$.

\section{Clinical evaluation of the patients revealed that}

Fifteen of the patients (50\%) had oral ulcers, 12 patients (40\%) had genital ulcers and all of our patients $(100 \%)$ had genital scars while only six patients $(20 \%)$ had erythema nodusum. Concerning eye manifestations, 16 patients $(53.3 \%)$ had diminution of vision, while 12 patients $(40 \%)$ had lacrimation, 13 patients $(43.3 \%)$ had photophobia, 13 patients $(43.3 \%)$ had redness and 7 patients $(23.3 \%)$ had eye pain. Only seven $(23.3 \%)$ of our patients had calf tenderness, 9 patients (30\%) had old DVT while 9 patients $(30 \%)$ had decrease in motor power, 14 patients $(46.7 \%)$ had arthralgia, three patients $(10 \%)$ had arthritis and two patients $(6.7 \%)$ had knee effusion. As regards pleural rub only one patient $(3.3 \%)$ was positive and no one had organomegaly or ascites. 


\section{Therapeutic measures of our patients}

All $(100 \%)$ of the patients were on Corticosteroids for a period ranging from $0.08-6$ years with a mean of $0.87 \pm 1.16$ on a dose ranging from $5-50 \mathrm{mg} /$ day with a mean of $19.3 \pm 1.28$.

Only fourteen (46.7\%) of the patients were on Azathioprine for a period ranging from $0.17-2$ years with a mean of $0.76 \pm 0.47$ on a dose ranging from $50-100 \mathrm{mg} /$ day with a mean of $78.6 \pm 2.57$.

\section{Laboratory evaluation of the patients revealed}

RBC count ranged from 3.17 to $6.11 \times 10^{6} / \mathrm{mm}^{3}$ with a mean of $4.5886 \pm 0.68867 \times 10^{6} / \mathrm{mm}^{3}$, Hemoglobin count ranged from 9.30 to $17.00 \mathrm{~g} \%$ with a mean of $14.20 \pm 2.08$ while Platelet count ranged from 192 to $394 \times 10^{3} / \mathrm{mm}^{3}$ with a mean of $295 \pm 47.55 \times 10^{3} / \mathrm{mm}^{3}$. W.B.C (PMNL) count ranged from 3.9 to $9.5 \times 10^{3} / \mathrm{mm}^{3}$ with a mean of $5.993 \pm 1.368 \times 10^{3} / \mathrm{mm}^{3}$ while (Lymphocytes) count ranged from 4 to $40 \times 10^{3} / \mathrm{mm}^{3}$ with a mean of $23.50 \pm 8.271 \times 10^{3} / \mathrm{mm}^{3}$. E.S.R ranged from 5.0 to $125.0 \mathrm{~mm} / 1^{\text {st }}$ with a mean of $40.2 \pm 26.2$.

Aspartate transaminase (AST) ranged from 9 to $34.0 \mathrm{U} / \mathrm{L}$ with a mean of $19.8 \pm 5.6$ while alanine transaminase (ALT) ranged from 8.0 to $49.0 \mathrm{U} / \mathrm{L}$ with a mean of $23.9 \pm 11.0$. Blood urea ranged from 10 to $42 \mathrm{mg} / \mathrm{dl}$ with a mean of $26.67 \pm 7.35$ while Serum creatinine ranged from 0.40 to $1.18 \mathrm{mg} / \mathrm{dl}$ with a mean of $0.75 \pm 0.20$. Cholesterol ranged from 143 to $270 \mathrm{mg} / \mathrm{dl}$ with a mean of $195 \pm 30.5$ while Triglycerides (TG) range from 51 to $194 \mathrm{mg} / \mathrm{dl}$ with a mean of $91.7 \pm 33.3$. Fasting blood sugar range from 69 to $100 \mathrm{mg} / \mathrm{dl}$ with a mean of $82.7 \pm 8.6$ while post prandial blood sugar ranged from 100 to $140 \mathrm{mg} / \mathrm{dl}$ with a mean of $118.7 \pm 1.028$.

\section{Ophthalmological examination reveled}

The number of the patients was 30 patients with 58 eyes, 30 right eyes and 28 left eyes as there were two patients had lost their left eyes. Twenty three eyes were normal. Two patients had bilateral conjunctivitis, where 6 patients had right sided conjunctivitis and 3 had left sided conjunctivitis. Seven patients had bilateral keratitis precipitances (KPs), while 10 patients had right sided KPs and 9 had left sided KPsTen patients had bilateral posterior synechia while 13 patients had right sided posterior synechia and 10 patients had left sided posterior synechia. One has bilateral lens affection while two of the patients had right sided cataract, two had left sided cataract and one has pigments on left lens. Three had bilateral cells in vitreous while five of the patients had right sided affection one of them was not seen and five patients had left sided affection one of them was not seen. Five patients had bilateral retinal vasculitis, while six patients had right sided retinal vasculitis and six had left sided retinal affection.

\section{Results of ocular doppler ultrasound on eye arteries}

Concerning readings of the right Ophthalmic artery (RT OA): PSV ranged from 6.7 to 57 with a mean of $27.5 \pm 13.5$, EDV ranged from 0.00 to 16.3 with a mean of $6.9 \pm 4.06$ and RI ranged from 0.63 to 1 with a mean of $0.76 \pm 0.08$ in the patients while it was 34 to 40 with a mean of $36.9 \pm 2.04,9$ to 12.3 with a mean of $10.6 \pm 1.3$ and 0.67 to 0.79 with a mean of $0.72 \pm 0.03$ in controls respectively.

A highly significant relations were found when PSV and EDV of RT( OA) artery were done comparing patients to controls (P-value were 0.001 and 0.000 respectively) and a significant relation was found when RI of RT( OA) artery was done comparing patients to controls (P-value 0.020).

Concerning results of the right central retinal artery (RT CRA): PSV ranged from 6.5 to 37.4 with a mean of $13.2 \pm 6.5$, EDV ranged from 1.6 to 11.8 with a mean of $4.4 \pm 2.1$ and RI ranged from 0.45 to 0.79 with a mean of $0.66 \pm 0.07$ in the patients while it was 9.4 to 14.9 with a mean of $11.8 \pm 1.52,2.9$ to 14.1 with a mean of $5.5 \pm 3.57$ and 0.61 to 0.72 with a mean of $0.66 \pm 0.03$ in controls respectively. Nonsignificant relations were found when PSV, EDV and RI of RT (CRA) were done comparing patients and controls (P-value were 0.288, 0.200 and 0.917 respectively).

Concerning results of the right posterior ciliary arteriey (RT PCA): PSV ranged from 6 to 45.7 with a mean of $13.8 \pm 8.3$, EDV ranged from 2.2 to 11.4 with a mean of $4.96 \pm 2.67$ and RI ranged from 0.49 to 0.76 with a mean of $0.63 \pm 0.06$ in the patients while it was 9.9 to 17.3 with a mean of $13.5 \pm 2.02,3.5$ to 6.1 with a mean of $5.2 \pm 0.99$ and 0.60 to 0.68 with a mean of $0.63 \pm 0.02$ in the controls respectively.

Non-significant relations were found when PSV, EDV and RI of RT (PCA) were done comparing patients to controls (P-value were $0.352,0.408$ and 0.409 respectively).

Concerning readings of the Left Ophthalmic artery (LT OA): PSV ranged from 7.7 to 65 with a mean of $26.4 \pm 12.3$, EDV ranged from 0.4 to 22.4 with a mean of $6.7 \pm 4.2$ and RI ranged from 0.62 to 0.95 with a mean of $0.75 \pm 0.08$ in the patients while it was from 32 to 43.8 with a mean of $38.7 \pm 3.17$, from 7.6 to 12.3 with a mean of $10.4 \pm 1.52$ and from 0.67 to 0.78 with a mean of $0.73 \pm 0.03$ in the controls respectively.

A highly significant relations were found when PSV and EDV of LT (OA) were done comparing patients to controls (P-value were 0.000 and 0.002 respectively) while no significant relation was found when RI of LT (OA) was done comparing patients to controls (Pvalue was 0.123 ).

Concerning results of the left central retinal artery (LT CRA): PSV ranged from 4.3 to 37.1 with a mean of $12.6 \pm 7.68$, EDV ranged from 1.6 to 25.8 with a mean of $4.8 \pm 4.9$ and RI ranged from 0.27 to 0.8 with a mean of $0.66 \pm 0.096$ in the patients while they were in the controls 8.5 to 14.9 with a mean of $12.5 \pm 1.9,2.6$ to 5.9 with a mean of $4.1 \pm 0.9$ and 0.61 to 0.71 with a mean of $0.67 \pm 0.037$ respectively. Non-significant relations were found when PSV, EDV and RI of LT (CRA) were done comparing patients to controls (P-value were 0.949, 0.466 and 0.883 respectively).

Concerning results of the left posterior ciliary arteries (LT PCA) in the patients: PSV ranged from 6.1 to 21.6 with a mean of $13.4 \pm 4.22$, EDV ranged from 1.9 to 7.32 with a mean of $4.70 \pm 1.65$ and RI ranged from 0.53 to 0.68 with a mean of $0.87 \pm 1.16$ while in the controls they were 10 to 15.4 with a mean of $13.5 \pm 1.57,3.2$ to 6.1 with a mean of $5.04 \pm 0.96$ and 0.59 to 0.69 with a mean of $0.63 \pm 0.03$ respectively.

Non-significant relations were found when PSV, EDV and RI of LT (PCA) were done comparing patients to controls (P-value were $0.872,0.386$ and 0.435 respectively) (Table 2) (Figures 1-6).

Correlations between age of the patients and (patient index score and average score), showed non-statistical significance as P-value were 0.580 and 0.735 respectively) (Figure7) (Figure 8).

Correlations between disease duration and (patient index score and average score) showed no statistical significance with P-value were 0.08 and 0.280 respectively (Figure 9) (Figure 10). 


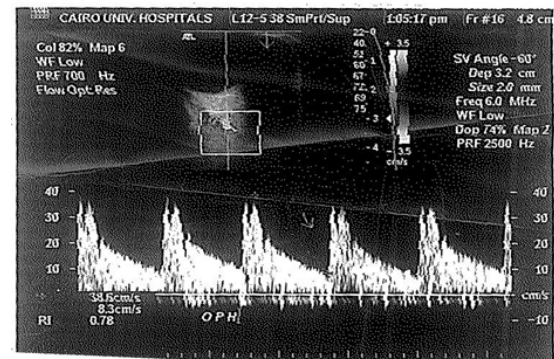

Figure I Color Doppler image showing Doppler parameters of a normal ophthalmic artery.

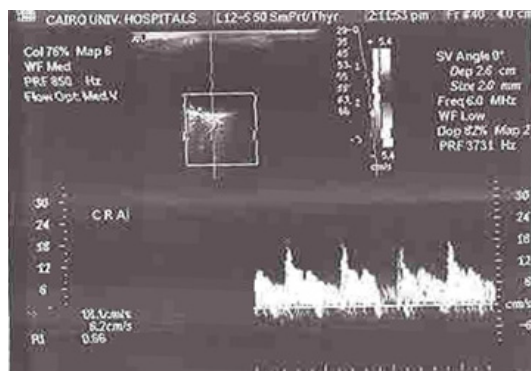

Figure 3 Color Doppler image showing Doppler parameters of a normal central retinal artery.

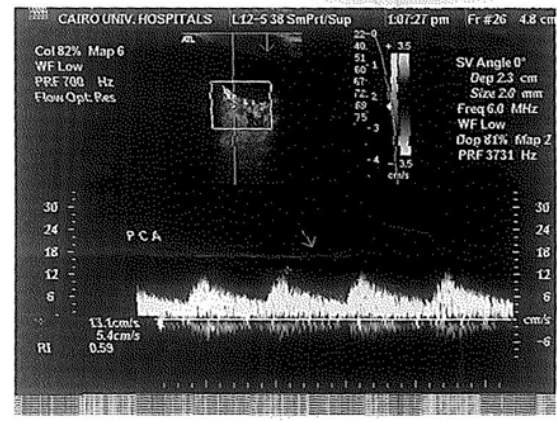

Figure 5 Color Doppler image showing Doppler parameters of a normal posterior ciliary artery.

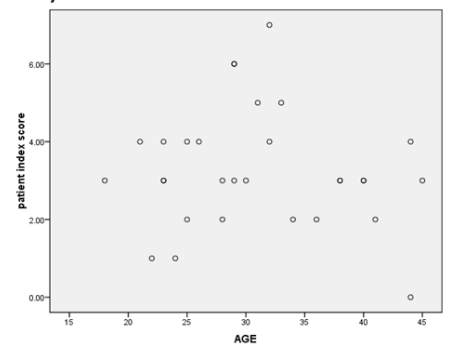

Figure 7 Correlations between Age and patient index score.

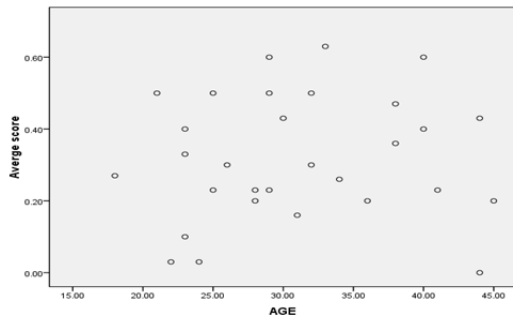

Figure 9 The correlation between disease duration and patient index score

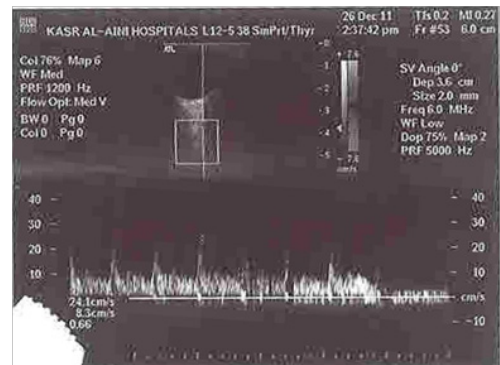

Figure 2 Color Doppler image showing Doppler parameters of the ophthalmic artery in a patient with ocular Behçet's disease.

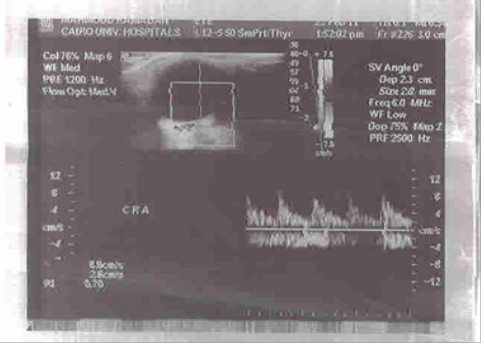

Figure 4 Color Doppler image showing Doppler parameters of the central retinal artery in a patient with ocular Behçet's disease.

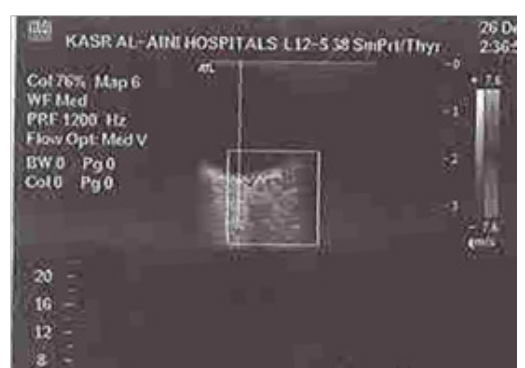

Figure 6 Color Doppler image showing Doppler parameters of the central posterior ciliary artery in a patient with ocular Behçet's disease.

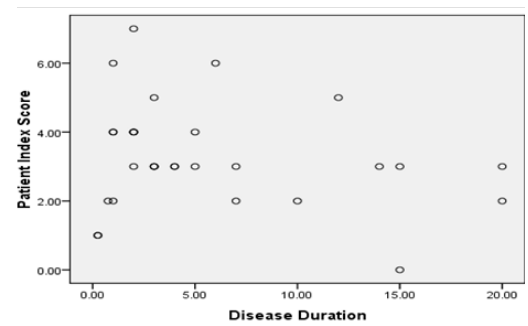

Figure 8 Correlations between age and average score.

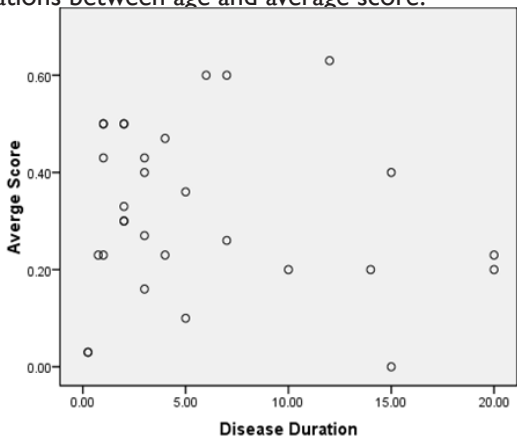

Figure 10 The correlation between disease duration and average score. 
Table 2 Results of ocular Doppler ultrasound over right (RT) and left (LT) sided arteries

\begin{tabular}{|c|c|c|c|c|c|c|c|c|c|c|}
\hline & \multicolumn{5}{|c|}{ RT sided arteries } & \multicolumn{5}{|c|}{ LT sided arteries } \\
\hline & & Range & Mean & S.D & P-value & & Range & Mean & S.D & P-value \\
\hline \multirow{2}{*}{ OA-PSV } & $\mathrm{P}$ & $6.7-57$ & 27.5 & 13.5 & \multirow[b]{2}{*}{0.001} & $\mathrm{P}$ & $7.7-65$ & 26.37 & 12.26 & \multirow{2}{*}{0.000} \\
\hline & $\mathrm{C}$ & $34-40$ & 36.9 & 2.04 & & $\mathrm{C}$ & $32-43.8$ & 38.73 & 3.17 & \\
\hline \multirow{2}{*}{ OA- EDV } & $\mathrm{P}$ & $0.0-16.3$ & 6.9 & 4.06 & \multirow[b]{2}{*}{0.000} & $\mathrm{P}$ & $0.4-22.4$ & 6.69 & 4.17 & \multirow{2}{*}{0.002} \\
\hline & $\mathrm{C}$ & $9-12.3$ & 10.6 & 1.33 & & $\mathrm{C}$ & $7.6-12.3$ & 10.36 & 1.52 & \\
\hline \multirow{2}{*}{ OA - RI } & $\mathrm{P}$ & $0.63-1$ & 0.76 & 0.08 & \multirow[b]{2}{*}{0.020} & $\mathrm{P}$ & $0.62-0.95$ & 0.75 & 0.08 & \multirow{2}{*}{0.123} \\
\hline & $\mathrm{C}$ & $0.67-0.79$ & 0.72 & 0.72 & & $\mathrm{C}$ & $0.67-0.78$ & 0.73 & 0.03 & \\
\hline \multirow{2}{*}{ CRA -PSV } & $\mathrm{P}$ & $6.5-37.4$ & 13.2023 & 6.53 & \multirow{2}{*}{0.288} & $\mathrm{P}$ & $4.3-37.1$ & 12.61 & 7.68 & \multirow{2}{*}{0.949} \\
\hline & $\mathrm{C}$ & $9.4-14.9$ & 11.8467 & 1.52 & & $\mathrm{C}$ & $8.5-14.9$ & 12.51 & 1.91 & \\
\hline \multirow{2}{*}{ CRA -EDV } & $\mathrm{P}$ & $1.6-11.8$ & 4.4297 & 2.13 & \multirow{2}{*}{0.200} & $\mathrm{P}$ & $1.6-25.8$ & 4.84 & 4.95 & \multirow{2}{*}{0.466} \\
\hline & $\mathrm{C}$ & $2.9-14.1$ & 5.5347 & 3.57 & & $\mathrm{C}$ & $2.6-5.9$ & 4.13 & 0.913 & \\
\hline \multirow{2}{*}{ CRA-RI } & $\mathrm{P}$ & $0.45-0.79$ & 0.6590 & 0.07 & \multirow{2}{*}{0.917} & $\mathrm{P}$ & $0.27-0.80$ & 0.66 & 0.096 & \multirow{2}{*}{0.883} \\
\hline & $\mathrm{C}$ & $0.61-0.72$ & 0.6573 & 0.03 & & $\mathrm{C}$ & $0.61-0.71$ & 0.67 & 0.037 & \\
\hline \multirow{2}{*}{ PCA -PSV } & $\mathrm{P}$ & $6-45.7$ & 13.82 & 8.35 & \multirow{2}{*}{0.352} & $\mathrm{P}$ & $6.1-21.6$ & 13.4 & 4.22 & \multirow{2}{*}{0.872} \\
\hline & $\mathrm{C}$ & $9.9-17.3$ & 13.53 & 2.02 & & $\mathrm{C}$ & $10-15.4$ & 13.5 & 1.57 & \\
\hline \multirow{2}{*}{ PCA -EDV } & $\mathrm{P}$ & $2.2-11.4$ & 4.96 & 2.67 & \multirow{2}{*}{0.408} & $\mathrm{P}$ & $1.9-7.32$ & 4.7 & 1.65 & \multirow{2}{*}{0.386} \\
\hline & $\mathrm{C}$ & $3.5-6.1$ & 5.16 & 0.99 & & $\mathrm{C}$ & $3.2-6.1$ & 5.04 & 0.96 & \\
\hline \multirow{2}{*}{ PCA - RI } & $\mathrm{P}$ & $0.49-0.76$ & 0.63 & 0.06 & \multirow{2}{*}{0.409} & $\mathrm{P}$ & $0.53-0.68$ & 0.87 & 1.16 & \multirow{2}{*}{0.435} \\
\hline & $\mathrm{C}$ & $0.6-0.68$ & 0.63 & 0.02 & & $\mathrm{C}$ & $0.59-0.69$ & 0.63 & 0.029 & \\
\hline
\end{tabular}

\section{C, Control; LT, Left; P, Patients; RT, Right; S.D, Standard deviation}

\section{Discussion}

Behçetdisease is a chronic systemic vasculitic disorder characterized by recurrent aphthous ulcers and intraocular inflammation. The clinical triad of uveitis with recurrent oral and genital ulcers bears the name of Hulusi Behçet, a Turkish dermatologist who described 3 patients who had this triad. ${ }^{7}$

In 1990, The International Study Group for Behçet's Disease proposed a separate set of diagnostic criteria for Behçet disease (3). Based on these criteria, a diagnosis of Behçet disease requires recurrent oral ulceration and at least 2 additional criteria, including recurrent genital ulcers, ocular lesions, skin lesions, and a positive pathergy test. ${ }^{7}$

The mean age at onset is in the third decade of life. However, when Behçet disease occurs in males aged 15-25 years, it takes a more serious form. ${ }^{8}$ The etiology of Behcet disease (BD) is unknown and although the definite pathogenic mechanism for the vascular lesions in $\mathrm{BD}$ remains unclear, endothelial dysfunction is thought to play an important role in the development of thrombophlebitis and venous thrombosis of vessels of any size. Patients with ocular involvement typically have a relapsing; remitting pan-uveitis and retinal vasculitis that affects all elements of the retinal vasculature. Retinal veins are primarily involved in the form of acute periphlebitis and occlusive vasculitis. ${ }^{8}$ Theories behind the pathogenesis of Behçet disease currently point toward an autoimmune etiology. Current research suggests that exposure to an infectious agent may trigger a crossreactive immune response. Proposed infectious agents have included herpes simplex virus (HSV), Streptococcus species, Staphylococcus species, and Escherichia coli, all of which commonly inhabit the oral cavity. ${ }^{9}$

Systemic involvement of multiple organs is observed in Behçet disease, rooted primarily in the development of vasculitis or vasculopathic lesions in the affected areas. These areas may demonstrate microscopic evidence of inflammatory tissue infiltration with both T cells and neutrophils. ${ }^{9}$
HLA-B51 has been shown to be more prevalent in Turkish, Middle Eastern, and Japanese populations, corresponding with a higher prevalence of Behçet disease in these populations. However, HLA-B51 has not been shown to affect the severity of symptoms. ${ }^{10}$

Behçet disease is characterized by severe recurrent attacks of intraocular inflammation. Anterior uveitis was present in 59\% of cases; posterior uveitis was present in $76 \%$ of cases; and pan-uveitis was present in $88.1 \%$ of cases. ${ }^{11}$

Doppler ultrasound is widely used as a noninvasive method for the assessment of the blood flow, both in the central and peripheral circulation. It may be used to estimate blood flow, to image regions of blood flow and to locate sites of arterial disease as well as flow characteristics and resistance of ophthalmic arteries ${ }^{12}$

Color Doppler ultrasound (CDU) is a reliable technique for evaluating orbital diseases and detecting early vasculitis, even if there weren't any clinical signs, so prompt treatment and follow up started early preventing serious complications which can lead to atrophy of the eye. In this study, all patients were subjected to full history taking, full clinical examination and routine laboratory investigations together with full ophthalmological examination. The radiologist, who was blinded to the clinical findings, examined the patients using (CDU).

In our study, results of right Ophthalmic artery (RT OA) were: the mean of PSV, EDV and RI were $27.5 \pm 13.5,6.9 \pm 4.1$ and $0.76 \pm 0.08$ in the patients respectively while they were $36.9 \pm 2.04,10.6 \pm 1.3$ and $0.72 \pm 0.03$ in the control group respectively.

A highly significant differences were found when PSV and EDV of RT (OA) were done comparing patients to controls (P- value were 0.001 and 0.000 respectively) and a significant difference was found when RI of RT (OA) was done comparing patients to controls (Pvalue 0.020 ).

While the results of the Left Ophthalmic artery (LT OA) were: the mean of PSV, EDV and RI were $26.4 \pm 12.3,6.7 \pm 4.2$ and $0.75 \pm 0.08$ 
in the patients respectively while they were $38.7 \pm 3.17,10.4 \pm 1.52$ and $0.73 \pm 0.03$ respectively in the control group.

A highly significant differences were found when PSV and EDV of LT (OA) were done comparing patients to controls (P-value were 0.000 and 0.002 respectively) while a non-significant difference was found when RI of LT (OA) was done comparing patients to controls (P- value was 0.123).

This agreed with the work done by Celebi and coworkers in 2000 as the mean of PSV of the OA in the patient group was significantly decreased when compared to the control group (P-value 0.02). ${ }^{13}$

Çaça et al., ${ }^{14}$ in 2004 divided their patients into two groups, group (I) included BD patients with ocular affection and group (II) included BD patients without ocular affection. Their results agreed with our work in that the PSV and EDV values of OA in group (I) were significantly lower when compared to those in the control group. ${ }^{12}$

Yilmaz \& $\mathrm{Akarsu}^{14}$ in 2006 divided their patients into two groups, group (1) included BD patients with ocular affection and group (2) included BD patients without ocular affection. Their results agree with our work in that the mean values of PSV and EDV were lower and RI of OA was higher in group( 1) than in group(2) and controls, but they did not reach statistical significance (P-value 0.05). ${ }^{14}$

Yanik et al., ${ }^{15}$ in 2006 divided BD patients into 3 groups, group (A) included patients with Behçet's disease in the active period, group(B) included patients with Behçet's disease in the inactive period and group $(\mathrm{C})$ included patients with Behçet's disease without ocular involvement. Their results are compatible with our results in that there were statistically significant differences between group A and the control group in PSV (P-value 0.01), EDV (P-value 0.01) and RI (P-value 0.01). ${ }^{15}$

Isik and coworkers in 2007 divided their patients into two groups, group (1) included BD patients with ocular affection and group (2) included BD patients without ocular affection. Their results are in agreement with our results in that there were statistically significant differences between group (2) and control subjects as regards PSV, EDV and RI of OA. ${ }^{15}$

The results of evaluation of the right central retinal artery (RT CRA) were: the mean of PSV, EDV and RI were 13.2 $\pm 6.5,4.4 \pm 2.1$ and $0.66 \pm 0.07$ respectively in the patients while they were $11.8 \pm 1.52$, $5.5 \pm 3.57$ and $0.66 \pm 0.03$ in the control group respectively.

Non-significant differences were found when PSV, EDV and RI of RT (CRA) were done comparing patients and controls (P-value were $0.288,0.200$ and 0.917 respectively).

While results of the left central retinal artery (LT CRA) were: the mean of PSV, EDV and RI were 12.6 $\pm 7.68,4.8 \pm 4.9$ and $0.66 \pm 0.09$ in the patients respectively while they were $12.5 \pm 1.9,4.1 \pm 0.9$ and $0.67 \pm 0.04$ respectively in the control group. Non-significant differences were found when PSV, EDV and RI of LT (CRA) were done comparing patients to controls (P-value were 0.949, 0.466 and 0.883 respectively).

Although the mean values of PSV and EDV were lower and RI of CRA was higher in patients than in controls, but they did not reach statistical significance.

Yilmaz \& $\mathrm{Akarsu}^{14}$ in 2006 agreed with our results as the PSV was lower and RI of CRA was higher in group(1) than in group (2) and in controls, but they did not reach statistical significance (P-value $0.05) \cdot{ }^{14}$
In contrast to our results they found that the EDV in the CRA were significantly lower in group (1)than in group (2) and in controls (P-value 0.037 and $\mathrm{P}$-value 0.045 , respectively). ${ }^{15}$

Yanik et al. ${ }^{15}$ in 2006 results agreed with our work in that there were non-significant differences in all Doppler parameters of CRA between Group C and the control group and the mean values of PSV was lower and RI of CRA was higher in (group A) than the controls, but they did not reach statistical significance (P-value 0.05 ).

However they didn't agree with our results as a highly significant difference was found between (group A) and control group concerning EDV value of CRA (P-value 0.001). ${ }^{15}$

Çaça et al., ${ }^{12}$ in 2004 agreed with our results in that there were non-statistical significant differences in the RI values of the CRA among the three groups. However they didn't agree with our results in that the mean PSV and EDV in the CRA were significantly lower in both $\mathrm{BD}$ patients groups than in healthy controls. Our results are in disagreement with Celebi et al., ${ }^{13}$ in 2000 and Isik et al., ${ }^{16}$ in 2007 who found that the mean values of PSV and EDV in the CRA were significantly lower in $\mathrm{BD}$ patients than in healthy controls.

Discrepancies between the results achieved in our study and studies done by others may be due to the difference in the age group of the selected patients, the difference in the number of patients, patients' characteristics (dividing their patients into patients with ocular affection and patients without ocular affection), Behçet's disease duration and the duration, severity and site of ocular vessel affection.

In our study, the results of the right posterior ciliary arteries (RT PCA) were: the mean of PSV, EDV and RI were 13.8 $\pm 8.3,4.9 \pm 2.67$ and $0.63 \pm 0.06$ respectively in the patients while they were $13.5 \pm 2.02$, $5.2 \pm 0.99$ and $0.63 \pm 0.02$ in the control group respectively.

Non-significant differences were found when PSV, EDV and RI of RT(PCA) were done comparing patients to controls (P-value were $0.351,0.408$ and 0.409 respectively). While results of the left posterior ciliary arteries (LT PCA) in the patients were: the mean of PSV, EDV and RI were $13.4 \pm 4.22,4.70 \pm 1.65$ and $0.87 \pm 1.16$ respectively while they were $13.5 \pm 1.57,5.04 \pm 0.96$ and $0.63 \pm 0.03$ respectively in the control group.

Non-significant differences were found when PSV, EDV and RI of LT (PCA) were done comparing patients to controls (P-value were $0.872,0.386$ and 0.435 respectively). Although the mean values of PSV and EDV were lower and RI of PCA was higher in patients than in controls, but they did not reach statistical significance.

The work done by Celebi et al., ${ }^{13}$ in 2000 agreed with our results in that there was non-significant difference in the mean of RI between the patients and the control group..$^{13}$

But they didn't agree with our results in that the mean values of PSV and EDV of PCA were significantly lower in the patient group than in the control group. ${ }^{13}$

Çaça et al., ${ }^{12}$ in 2004 divided PCA into temporal (TPCA) and nasal (NPCA).They agreed with our results in that the PSV in NPCA and EDV in TPCA in group (II) were not significantly different when compared with those in the control group. The EDV, RI values of NPCA and RI values of the TPCA were not significantly different among the three groups. ${ }^{12}$

However in contrast to our results they found that there were highly significant differences between group (I) and the control group in each of the following: PSV (P-value 0.007) in the NPCA, PSV (P-value 0.001) and EDV (P-value 0.003) in the TPCA. ${ }^{12}$ 
Yanik et al., ${ }^{15}$ in 2006 divided PCA into temporal (TPCA) and nasal (NPCA). The results agreed with our work as there were nonsignificant differences in all Doppler parameters between group $(\mathrm{C})$ and the control group.

But they didn't agree with our work in that there were statistically significant differences between $\operatorname{group}(\mathrm{A})$ and the control group in each of the following: PSV (P-value 0.01) in TPCA and PSV (P-value $0.05)$ in NPCA and highly statistically significant differences in each of the following: EDV (P-value 0.001), and RI (P-value 0.001) in the TPCA, and EDV (P-value 0.001), and RI (P-value 0.001) in the NPCA. ${ }^{15}$

Yilmaz \& $\mathrm{Akarsu}^{14}$ in 2006 agreed with our results in that PSV of PCA didn't show statistically significantdifference when group(1)was compared with group(2) patients and controls. ${ }^{14}$

But in contrast to our results they found that group(1) patients had significantly lower EDV and higher RI than did group (2) patients and controls in their PCA. ${ }^{14}$

Differences between the results achieved in our study and studies done by others may be due to the number of recurrences of ocular affection, the site of CDU sampling (as they divided the PCA into temporal and nasal), difference in the age group of the selected patients and patients' characteristics.

In our work we studied the correlation between the age of the patients and (patient index score and average score) as a measure of disease activity and it was found to have non-statistical significance as P-values were 0.580 and 0.735 respectively. Correlation between disease duration and (patient index score and average score) showed non-statistical significance as P-values were 0.08 and 0.280 respectively. This could be explained by the relatively smaller number of patients, shorter disease duration and younger ages of our patients. There are no published results to compare our values with, so further long term studies with larger number of patients and longer disease duration are needed to assess the relation of age and disease duration with disease activity.

\section{Conclusion}

Behçet's disease patients with ocular involvement have lower CRA, PCA and OA blood flow velocities than healthy control. CDU is helpful in early diagnosis of ocular Behçet's disease activity as it is a widely used, easy-to-perform and accurate method.

\section{Acknowledgements}

None.

\section{Conflict of interest}

Author declares there is no conflict in publishing the article.

\section{References}

1. Kaçmaz O, Kempen JH, Newcomb C. The Systemic Immunosuppressive Therapy for Eye Diseases: Cohort Study Group. Am J Ophthalmol. 2008;146(6):828-836.
2. Nagaoka T. Physiological mechanism for the regulation of ocular circulation. Nippon Ganka Gakkai Zasshi. 2006;110(11):872-8.

3. Criteria for diagnosis of Behçet's disease. Lancet. 1990;335(8697):10781080 .

4. Bhakta BB, Brennan P, James TE, et al. Behcet's disease: evaluation of a new instrument to measure clinical activity. Rheumatology. 1999;38(8):728-33.

5. Popa ED, Stanila A. Ocular blood flow assessment by color Doppler imaging in glaucoma. Acta Medica Transil Vanica. 2010;2(4):223-224.

6. Harrisa A, Chung HS, Ciulla TA, et al. Progress in measurement of ocular blood flow and relevance to our understanding of glaucoma and age-related macular degeneration. Progress in Retinal and Eye Research. 1999;18(5):669-687.

7. Houman MH, Hamzaoui K. Promising new therapies for Behcet's disease. Eur J Intern Med. 2006;17(3):163-9.

8. Dursun A, Durakbasi-Dursun HG, Dursun R, et al. Angiotensinconverting enzyme gene and endothelial nitric oxide synthase gene polymorphisms in Behçet's disease with or without ocular involvement. Inflammation research. 2009;58(7):401-405.

9. Keino H, Okada AA, Watanabe T, et al. Decreased ocular inflammatory attacks and background retinal and disc vascular leakage in patients with Behcet's disease on infliximab therapy. Br Ophthalmol. 2011;95(9):1245-50.

10. Krause I, Yankevich A, Fraser A, et al. Prevalence and clinical aspects of Behcet's disease in the north of Israel. Clin Rheumatol. 2007;26(4):55560 .

11. Torres RM, Yanez B, Herreras JM, et al. Ocular Behcet disease. Retrospective study. Arch Soc Esp Oftalmol. 2004;79(12):599-603.

12. Çaça I, lu HN, Ünlü K, et al. Color Doppler Imaging of Ocular Hemodynamic Changes in Behcet's Disease. Jpn J Ophthalmol. 2004;48:101-105.

13. Celebi S, Akfýrat M, Celebi H, et al. Color Doppler ultrasonography in ocular Behcet' disease. Acta Ophthalmol. 2000;78:30-33.

14. Yilmaz S, Akarsu C. Changes in cerebral and ocular hemodynamics in Behçet's disease assessed by color-coded duplex sonography, European Journal of Radiology. 2006;58(1):102-109 .

15. Yanik B, Conkbayir I, Berker N, et al. Doppler ultrasonography findings in ocular Behçet’s disease. Clinical Imaging. 2006;30(5):303-308.

16. Isik C, Yagci B, Yildirim C, et al. Orbital color Doppler imaging in Behçet's disease with or without ocular involvement. Int Ophthalmol. 2007:27(1):37-42. 